\section{Psychosomatik in der Augenheilkunde}

In der Medizin vollzieht sich in den letzten Jahren ein bedeutender Wandel.

Neben den Erfolgen unseres rein biomedizinischen Krankheitsverständnisses nimmt die Anzahl der chronischen Beschwerden immer mehr zu.

Diese in ihrer Ätiologie weitgehend ungeklärten Erkrankungen lassen das biomedizinische Modell bald an ihre Grenzen stoßen. Die Diagnosen werden daher meist mit dem Attribut: „essentiell", „idiopathisch“, „endogen“, „primär" versehen.

Lange wurde der Arzt nur dann als wissenschaftlich Handelnder eingestuft, seine Medizin als wissenschaftlich korrekt angesehen, wenn er sich mit Körperprozessen befasste, nicht aber, wenn er den Patienten als Menschen in den Mittelpunkt stellte. Dabei wird aber jeder länger praktizierende Mediziner feststellen, dass wir in unserem Beruf auf zwei Ebenen handeln, auf der des Beobachtens und der der Beziehung,

Nicht länger können wir uns der Erkenntnis verschließen, dass psychosoziale Einflüsse auf Entstehung, Verlauf und Endzustand von Krankheiten ebenso wichtige Probleme für die Heilkunde darstellen, wie die Einflüsse physikalischer, chemischer und biologischer Faktoren. Wobei Immunsystem und Neuroendokrinologie als wichtigste Schnittstellen anzusehen sind.

Die Psychosomatik vollzieht mit ihrem Verständnis der Auswirkungen von Psyche auf Soma und Soma auf Psyche den Übergang des bio-mechanischen Modells auf das biopsycho-soziale Modell.

Aus diesen Überlegungen wurde 1999 von mir die österreichische psychosomatische Gesellschaft in der Augenheilkunde (ÖGPA) gegründet.

Das Ziel der Vereinigung ist es:

- Koordination von Aus- und Weiterbildung, Forschung und Praxis der psychosomatischen Medizin in der Augenheilkunde, Unterstützung des Transfers von Forschungsergebnissen in die klinische Praxis;

- Förderung von nationalen und internationalen Kontakten auf dem Gebiet der Psychosomatik durch Tagungen, Seminare und Workshops;

- besonderes Anliegen ist es, eine Plattform für fachübergreifende Themen, interdisziplinäre Therapiekonzepte und den Dialog zwischen Ärzten verschiedener sFachrichtungen zu bilden.

Prim. Dr. Roman Rybiczka www.psychosomatik-augen.at dr.rybiczka@augen-wienwest.com 